\author{
Military Technical College \\ Kobry El-Kobbah, \\ Cairo, Egypt.
}

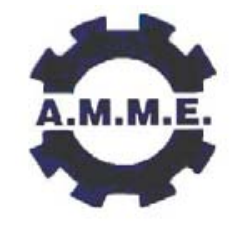

\title{
EXPERIMENTAL AND NUMERICAL STUDY OF POWDER METALLURGY PERFORMANCE FOR AUTOMOTIVE APPLICATIONS
}

\author{
HRAIRI $^{*}$ M., HUSSIN ${ }^{* *}$ M.A. and RAVI ${ }^{* *}$ N.M.F
}

\begin{abstract}
Powder metallurgy (P/M) processes using sintering are able to form net-shaped products and have been used widely in the production of automobile parts to improve productivity. In this work, an experimental and numerical study on the substitution by $\mathrm{P} / \mathrm{M}$ in automotive parts was carried out. Aluminium alloy A356 powder was used in the experimental part while stainless steel 316L powder was the material of choice in the numerical study. The A356 powder was shaped by a uniaxial press then sintered at 520 ${ }^{\circ} \mathrm{C}$. The density, compressive strength, and hardness of the produced part were evaluated and compared to the values for the same material produced by the permanent mould cast process. In the simulation component of this study, ANSYSTM Finite Element software was used to model an axisymmetric flanged part via the LSDYNA ${ }^{\mathrm{TM}}$ module using the geological Cap material model. The effect of density distribution in the part was analyzed through the plastic strain that was generated. The result was compared to the experimental density map.
\end{abstract}

\section{KEY WORDS}

Powder metallurgy, compaction, sintering, simulation, finite element method, density, hardness, compressive strength, automotive engineering

* Assistant professor, Dept. of Mechanical Engineering, IIUM, Kuala Lumpur, Malaysia

** Graduate student, Dept. of Mechanical Engineering, IIUM, Kuala Lumpur, Malaysia 


\section{NOMENCLATURE}

$\begin{array}{ll}C & \text { the elastic constitutive tensor } \\ J_{1} & \text { first invariant of the stress } \\ J_{2 d} & \text { second invariant of the deviatoric stress tensor } \\ T & \text { maximum hydrostatic tension sustainable by the material. } \\ X(\kappa) & \text { the intersection of the cap surface with the } \mathrm{J}_{1} \text { axis } \\ \kappa & \text { hardening parameter } \\ \varepsilon^{e} & \text { elastic strain } \\ \varepsilon^{p} & \text { plastic strain. } \\ \varepsilon_{v}^{p} & \text { volumetric plastic strain } \\ \sigma & \text { the stress } \\ \rho_{0} & \text { initial density } \\ \rho & \text { final density } \\ \lambda_{k} & \text { the plastic consistency parameter for surface } \mathrm{k} .\end{array}$

\section{INTRODUCTION}

Powder Metallurgy (P/M) is on the leading edge of new manufacturing processes for improved product quality and productivity. It is a cost-effective method of forming precision net-shape metal components that allows for more efficiently designed consumer and industrial products.

The basic process of P/M consists of mixing, filling, compacting, sintering, and finishing with secondary operations [1]. Mixing or blending the powders normally incorporates an organic substance to act as a lubricant during the next step. This mix is tested to ensure conformity with predetermined standards. Then, the powder mix is loaded into a suitable die or mould and consolidated by the application of pressure into what is referred to as a compact. Compacts must have sufficient strength to permit handling without fracture or crumbling, but are in no way strong enough for any engineering application. The compacts are then sintered, generally in a protective atmosphere, to cause the particles to weld together thus generating the strength required for use. Finally, secondary operations are carried out if necessary.

$\mathrm{P} / \mathrm{M}$ components are used in a variety of markets, with the automotive industry being the predominant one, consuming approximately $70 \%$ of the ferrous products the industry produces annually. The P/M industry has enjoyed the benefits of increased content in light vehicles. P/M technology provides total cost savings, unique properties, reliability, special materials and design capabilities, quality, and increased performance. Automotive design engineers have been converting castings, forgings, and stamping into $\mathrm{P} / \mathrm{M}$ when designing new engines and transmissions. Typical P/M components in modern engines and transmissions include main bearing caps, planetary carries, chain sprockets, and gears.

The $P / M$ industry continues to invest heavily in new technology to improve the properties of $\mathrm{P} / \mathrm{M}$ products. One of the major goals for the conventional $\mathrm{P} / \mathrm{M}$ industry is 
to increase the density to match the properties of wrought materials. New materials containing chromium, silicon and other non-conventional alloy systems were developed for higher performance. New lubricant systems are being developed for higher densities and enhanced properties, closing the gap with wrought materials [2].

In the compaction process of metal powders into rigid dies, one of the most common problems is cracking during either the compaction stage or ejection from the die. Therefore, the finite element based simulation is considered to be a design tool for powder metallurgy parts, as well as for compaction tooling, as this method allows the prediction of any kind of stresses and density distributions of the pressed compact prior to the actual tooling manufacturing activity [3].

In the current work, the advantages of the P/M process over a conventional mold cast process that can be applied to produce automotive parts is being investigated. To begin with, the mechanical properties of the A356 alloy such as the compressive strength, Vickers hardness, and relative density, have been determined experimentally and compared to those of a component manufactured using the permanent mold cast process. Then, ANSYS LS-DYNA ${ }^{\mathrm{TM}}$ was used to simulate the compaction process using the geological Cap material model. The numerical results were then compared to the experimental results.

\section{EXPERIMENTAL PROCEDURE}

The A356 powder was shaped and compacted at a pressure of 3 tons (2000 psi) for 10 minutes using a uniaxial press. Cylindrically shaped specimens of $15.93 \mathrm{~mm}$ diameter were produced as shown in Fig. 1. The green compacts were sintered for 10 hours at $520^{\circ} \mathrm{C}$. The heating rate was set at $0.27^{\circ} \mathrm{C} / \mathrm{sec}$. The part density was measured using an electronic densimeter model MS-200S. The Archimedes principle was used to quantify the density of the samples. The hardness of the compacts was evaluated as a function of the applied pressure and sintering time. Vickers microhardness measurements were carried out on specimens using a digital microhardness tester at five points on the specimen. A compression test was used to evaluate the compression strength at room temperature. The specimens were tested using the Universal Testing Machine INSTRON 5582.

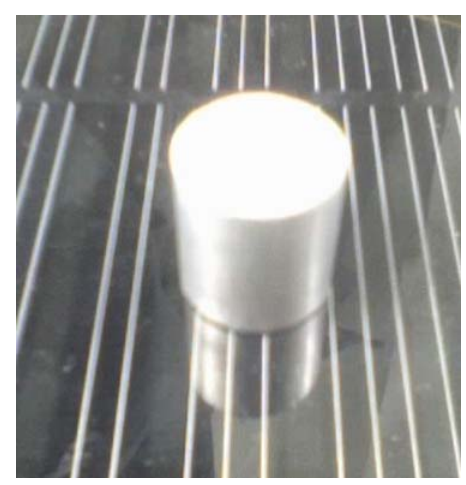

Fig. 1. Compacted A356 aluminum alloy 


\section{NUMERICAL STUDY}

\section{Material Model}

The basis for successful predictions of shape distortions or the tendency to develop cracks during pressing, unloading, or ejection of the part from die is a reliable constitutive model for describing the mechanical response of the powder. Thus, in this analysis, the Geological Cap Material Model has proven to be the most suitable material model since it has the ability to control the amount of dilatancy produced under shear loading and to model plastic compaction.

The Geological Cap model was originally developed for geological materials. This model has been used to simulate cold die compaction of metal powders and it has shown flexibility in modeling all compaction stages. This model can also be used to simulate the compaction of powders, starting from the loose state up to very high density levels. The first numerical simulations using the Cap model were based on the algorithm proposed by Sandler and Rubin [4]. This algorithm was found not to be fully coherent with the principles of plastic consistency and associativity of the flow rule and was later corrected for these limitations [5]. Hofstetter et al. then proposed an improved formulation of the Cap model yield functions in order to ensure a better numerical stability of the model. They also derived a consistent expression of the algorithmic elastoplastic tangent moduli. The use of this algorithmic moduli in place of the so-called continuum moduli helps to preserve the quadratic rate of convergence when a NewtonRaphson scheme is used for solving the FE problem [6]. As shown in Fig. 2, the Cap model consists of three surfaces in pressure $\sqrt{J_{2 d}}-J_{1}$ space, surface $f_{1}$ is the failure envelope, $f_{2}$ is the Cap surface, and $f_{3}$ is the tension cutoff that intersects in a nonsmooth manner $[6,7]$.

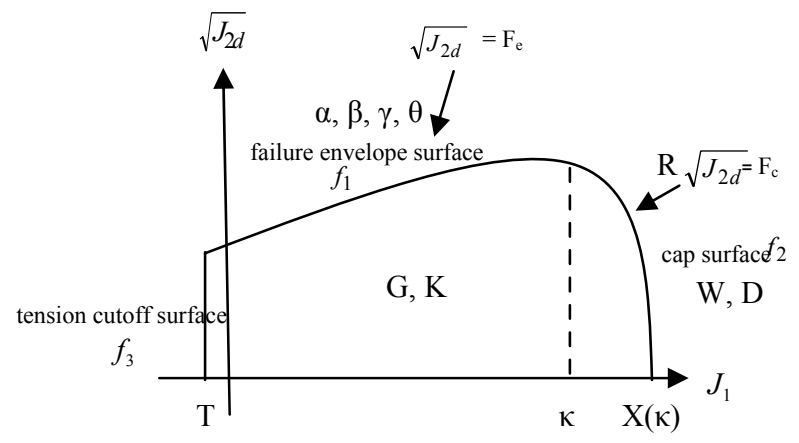

Fig. 2. Surface of the Two-invariant Cap model

The functional form of the failure envelope surface is given by:

$$
f_{1}=\sqrt{J_{2 d}}-\min \left(\alpha-\gamma e^{-\beta J_{1}}+\theta J_{1},\left|X\left(\kappa_{n}\right)-L\left(\kappa_{n}\right)\right|\right)
$$

where $X(\kappa)$ and $L(\kappa)$ are defined by: 


$$
\begin{aligned}
& X(\kappa)=\kappa+R\left(\alpha-\gamma e^{-\beta \kappa}+\theta \kappa\right) \\
& L(\kappa)= \begin{cases}\kappa & \text { if } \kappa>0 \\
0 & \text { if } \kappa \leq 0\end{cases}
\end{aligned}
$$

This failure envelope surface is fixed in $\sqrt{J_{2 d}}-J_{1}$ space, and therefore does not harden unless kinematic hardening is present. The cap surface $f_{2}$ is given by:

$$
f_{2}=\sqrt{J_{2 d}}-\frac{1}{R} \sqrt{[X(\kappa)-L(\kappa)]^{2}-\left[J_{1}(\kappa)-L(\kappa)\right]^{2}}
$$

The hardening parameter $\kappa$ is related to the plastic volume change $\varepsilon_{v}^{p}$ through the hardening law:

$$
\varepsilon_{v}^{p}=W\left(1-e^{-D\left(X(\kappa)-X_{0}\right)}\right)
$$

Geometrically, $\kappa$ is seen in the figure as the $J_{1}$ coordinate of the intersection of the Cap surface and the failure surface.

The hardening of the Cap will be modeled through the hardening rule relating the hardening state variable to the volumetric plastic strain in a form suggested by the results of the classical hydrostatic pressing test.

Finally, the function $f_{3}$ of the tension cutoff surface is given by:

$$
f_{3}=T-J_{1}
$$

The elastic domain in $\sqrt{J_{2 d}}-J_{1}$ space is then bounded by the failure envelope surface above, the tension cutoff surface on the left, and the cap surface on the right.

An additive decomposition of the strain into elastic and plastic parts is assumed:

$$
\varepsilon=\varepsilon^{e}+\varepsilon^{p}
$$

Stress is found from the elastic strain using Hooke's law,

$$
\sigma=\mathbf{C}\left(\varepsilon-\varepsilon^{p}\right)
$$

For the geological Cap model, and as shown in Fig. 2, a number of parameters must be chosen to represent a particular material. These are generally based on experimental data. The parameters $\alpha, \beta, \theta$ and $\gamma$ are usually evaluated by fitting a curve through failure data taken from a set of triaxial compression tests. The parameters $W, D$, and $X_{0}$ define the Cap hardening law. The value $W$ represents the void fraction of the uncompressed sample and $D$ governs the slope of the initial loading curve in hydrostatic compression. The value of $R$ is the ratio of major to minor axes of the quarter ellipse defining the Cap surface. $\mathrm{G}$ and $\mathrm{K}$ represent the elastic moduli of the material and are expressed as a function of the density. 


\section{Simulation Approach}

The part to be compacted is an axisymmetric flanged part made of $316 \mathrm{~L}$ stainless steel. The initial dimensions of the die cavity, the expected final dimensions of the pressed part, as well as the tooling displacements are given in Fig. 3.

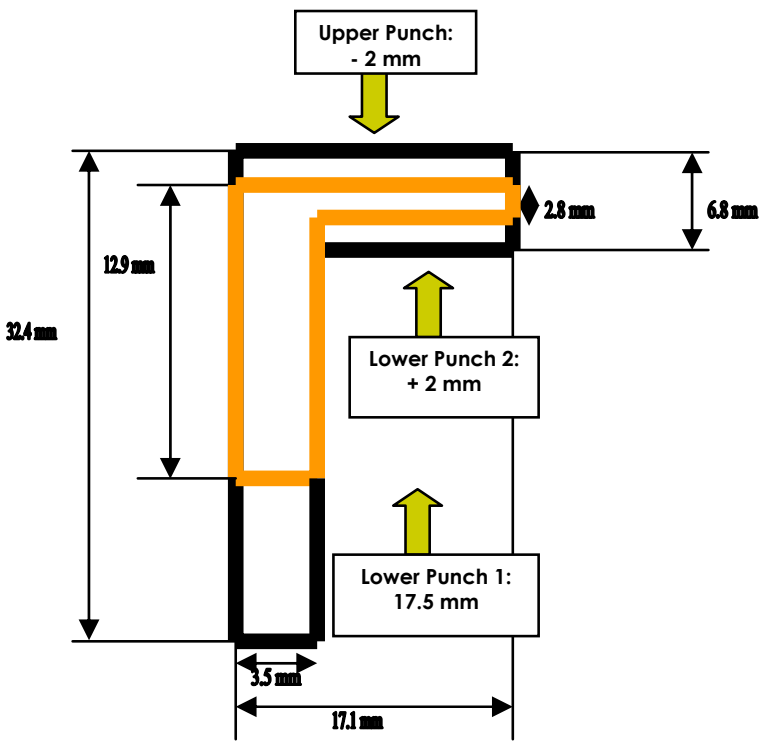

Fig. 3. Axisymmetric two level part before and after compaction

The parameters for $316 \mathrm{~L}$ stainless steel powder used in this simulation are taken from [8]. The ANSYSTM output file (.k file) is entered into LS-Prepost before changing the parameters' values in accordance to the standard table of units. The LS-Prepost is a valuable tool for ANSYSTM that provides accurate external values rather than solving with ANSYSTM itself. This is done because LS-Prepost has fewer limitations. The analysis is done by the LS-DYNATM solver which is known to provide accurate results which are key for any complex analysis. LS-DYNATM has also been used as a solver for other software analysis tools such as Virtual Proving Ground (VPG).

\section{RESULTS AND DISCUSSIONS}

\section{Experimental Results}

A356 alloy is used in automotive transmission cases, water cooled cylinder blocks, pistons, connecting rods, and also other moderate strength components [9]. Pistons are made from materials having properties such as high compressive strength and high hardness in order to handle the high temperature and pressure produced in the cylinder while combustion strokes take place. Pistons also need to have high corrosive resistance because they are exposed to highly combustible air and fuel mixtures. The reciprocating piston that goes up and down in motion also needs to be very wear resistant in order to maintain a longer life cycle of the piston. Based on the experimental study conducted in this work, the A356 produced parts using the P/M process seem to have better mechanical properties than similar parts produced using the conventional 
permanent mold cast process. This A356 material can also be applied to make the engine valves which require very similar characteristics to the piston.

Porosity is one of the main structural parameters that dictate the quality of P/M products. In fact, the mechanical properties of P/M parts are usually degraded by the presence of pores. Strength is the first concern when dealing with most structural P/M components. At a given porosity, smooth pores give the greatest strength. Relative density, hence porosity, is a critical factor in powder compacts as it significantly affects densification behavior as well as elastic and mechanical properties. The average relative density for A356 compacts was experimentally determined to be $92.6 \%$.

Modifications to the sintering cycle, such as a longer time or higher temperature, could also improve strength. The A356 part produced by the P/M process seems to give better mechanical properties in compressive strength and hardness values than components produced using the permanent mold cast process. The obtained results for the compressive strength were $260.97 \mathrm{MPa}$ and $185 \mathrm{Mpa}$ respectively. As for the Vickers hardness values, they were an average of 92.672 for the P/M part compared with 90 for the permanent mold cast part.

\section{Numerical Results}

From the simulation, we obtained the displacement and the volumetric plastic strain data that is used to determine the density distribution. The general equation of density that corresponds to the volumetric plastic strain is:

$$
\rho=\rho_{0} e^{\varepsilon_{v}^{p}}
$$

Therefore, from the result obtained, calculation of density is done for maximum, intermediate, and minimum values of $\varepsilon_{v}^{p}$. The predicted density values will be compared to the density values obtained from an experimental density map. The different density distribution maps are given in Fig. 4. From this figure, it can be noted that many of the density obtained in LS-DYNA ${ }^{\mathrm{TM}}$ correspond well to the experimental density map. For example, for the maximum value of density, LS-DYNA ${ }^{\mathrm{TM}}$ data shows $89.5 \%$ while the experimental data show $87 \%$. Thus, it shows that the bottom part of the flange for LS-DYNATM experienced the larger loads as it is displaced to $17.5 \mathrm{~mm}$. In brief, the larger the load displacement or the higher the compaction, the higher the density value that results from it.

On the other hand, for the intermediate density value, LS-DYNATM data shows $87.7 \%$ while the experimental data shows $84 \%$. Nevertheless, for the minimum value of density, LS-DYNA ${ }^{\mathrm{TM}}$ data shows $86 \%$ while the experimental data shows $81 \%$. In addition, the error percentage for time and displacement obtained are $1.25 \%$ and $1.03 \%$ respectively. It is a limitation within LS-DYNATM to have to specify a fixed value of $R$ whereas the latter should be defined as a function of the compact density [10-12]. 


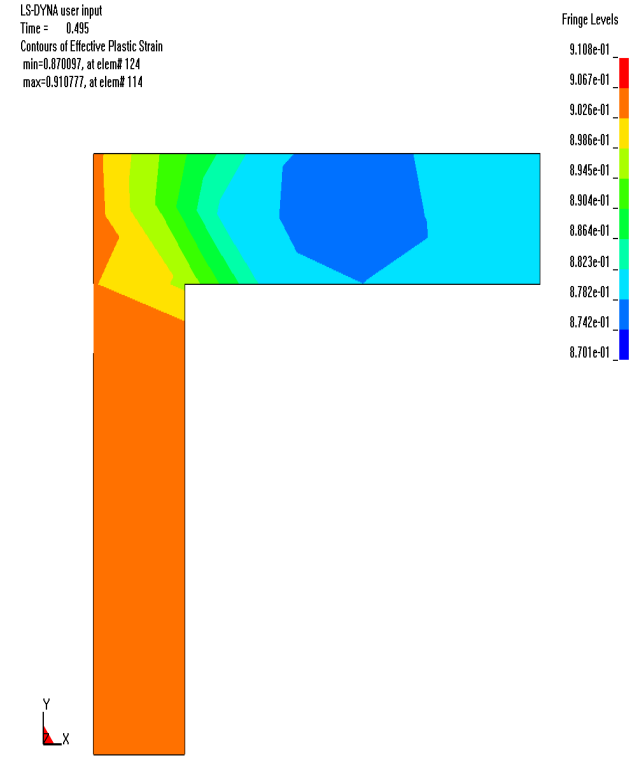

a)

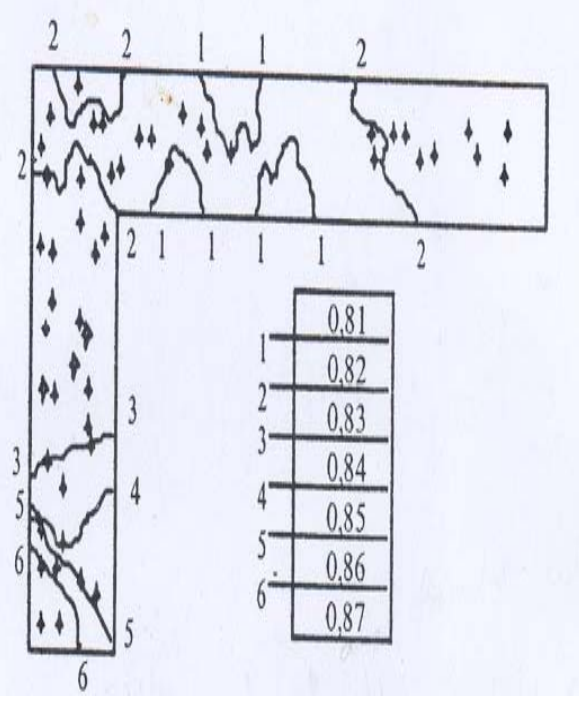

b)

Fig. 4. Map of density distribution a) LS-DYNATM FE results b) Experimental results

\section{CONCLUSION}

Based on the results obtained from the current study, the A356 aluminum alloy P/M compact possesses good mechanical properties. The achieved relative density of $92 \%$ was within industry standards. In addition, the compressive strength of $260.97 \mathrm{MPa}$ and the Vickers hardness average of 92.672 were also comparable to permanent mold cast parts.

Numerically, it has been shown that the predicted density distribution using FE software ANSYS ${ }^{\mathrm{TM}}$ correlate well with those results obtained experimentally. The difference in the value of density is related to the material model used in which LS-DYNA ${ }^{\mathrm{TM}}$ treated the aspect ratio $\mathrm{R}$ as a constant value for the whole density distribution whereas it should be evaluated as a function of density.

\section{REFERENCES}

[1] German R.M., Powder Metallurgy Science. $2^{\text {nd }}$ Edition , MPIF, Princeton N.J., (1984).

[2] Ryuichiro G., "Powder Metallurgy Growth in the Automotive Market”, Business Briefing, vol. 6, pp. 44-46, (2003).

[3] Crawford J. and Lindskog P., "Constitutive Equations and their role in the Modeling of the Cold Pressing Process", Scandinavian Journal of Metallurgy, vol.12, pp. 271-281, (1983).

[4] Sandler I. S. and Rubin D., "An Algorithm and a Modular Subroutine for the Cap Model", International Journal for Numerical and Analytical Methods in Geomechanics, vol.3, pp. 173-186, (1979).

[5] Simo J.C., Ju J.W., Pister K.S., and Taylor R.L., "Assessment of Cap Model: 
Consistent Return Algorithms and Rate Dependent Extension", Journal of Engineering Materials, vol. 114-2, pp. 191-218, (1988)

[6] Hofstetter G., Simo J.C., and Taylor R.L.,"A Modified Cap Model: Closest Point Solution Algorithms", Computers and Structures, vol. 46, no 2, pp. 203-214, (1993).

[7] Dimaggio F. and Sandler I., "Material Model for Granular Soils", Journal of the Engineering mechanics Division, pp. 935-950, (1971).

[8] Chtourou H., Gakwaya A., Guillot M., and Hrairi M., "Implementing a Cap Material Model for the Compaction of Metal Powder Compaction", Symposium on the Net Shape Processing of Powder Materials, ASME International Mechanical Engineering Congress and Exposition, AMD-Vol. 216, San Francisco, USA, (1995).

[9] http://www.efunda.com/

[10] Chtourou H., Gakwaya A., Guillot M., and Hraïri M., "Finite Element Simulation of the Rigid Die Compaction of Metal Powder Components," Symposium of the CSME 96 Forum, Hamilton, Canada, (1996).

[11] SAS IP Inc. ANSYS Training Manual. 1st Ed., (2004).

[12] LS-DYNA Keyword User Manual, Version 971, LSTC, (2007). 